\title{
Nanoscale morphology and electronic coupling at the interface between indium tin oxide and organic molecular materials
}

\author{
Andrea Lorenzoni, ${ }^{a}$ Adriano Mosca Conte, ${ }^{b}$ Alessandro Pecchia ${ }^{b}$ and Francesco Mercuri ${ }^{* a}$
}

The correlation between nanoscale morphology and charge injection rates at the interface between an organic semiconductor layer and a transparent metal oxide electrode was investigated by integrating molecular dynamics simulations with electronic structure calculations. The simulation approach proposed has been applied to the analysis of the hole injection mechanism at the interface between an amorphous layer of tris[(3-phenyl-1H-benzimidazol-1-yl-2(3H)-ylidene)1,2-phenylene]Ir (DPBIC), a hole transport and emitter molecule, and the surface of indium tin oxide (ITO), a material commonly used as anode in OLEDs. The link between interface morphology and charge injection was investigated by implementing a two-step, top-down simulation approach. Namely, nanoscale molecular aggregation phenomena at the organic/electrode interface were first assessed by molecular dynamics simulations, mimicking different processing conditions, and followed by density functional theory calculations of the electronic coupling between molecular levels and the manifold of electrode states involved in the charge injection process. The correlation between structural parameters and electronic coupling suggests a significant role of specific molecule/electrode configurations on charge transport processes at the interface, resulting in a broad distribution of charge injection rates, and highlights the link between molecular structure, nanoscale aggregation and processing in the realization of heterointerfaces for efficient charge injection in organic electronic devices.

\footnotetext{
${ }^{a}$ Istituto per lo Studio dei Materiali Nanostrutturati (ISMN), Consiglio Nazionale delle Ricerche (CNR), Via P. Gobetti 101, 40129 Bologna, Italy. Fax: +39 051 6398540 ; Tel: +39051 639 8518; E-mail: francesco.mercuri@cnr.it

${ }^{b}$ Istituto per lo Studio dei Materiali Nanostrutturati (ISMN), Consiglio Nazionale delle Ricerche (CNR), Via Salaria Km 29,300, 00015 Monterotondo Stazione (Roma), Italy.
} 


\section{Introduction}

The interface between metal electrodes and organic materials plays a central role in the development of novel functional devices, including organic light-emitting diodes (OLEDs), organic thin-film transistors (OTFTs) and organic photovoltaics (OPV). 1. $1-7$ In particular, the injection of charges from an electrode into an organic thin film is one of the processes that crucially affects the global functioning of OLEDs and OTFTs. ${ }^{811}$ A quantitative and realistic description of the phenomena related to charge injection at the metal-organic interface, however, is still a very challenging task, requiring the interplay of several models for the simulation of interface morphology, electronic coupling and ultimately injection dynamics. The interface morphology can be predicted by molecular dynamics simulations, typically parameterized using density-functional calculations on small subsystems. $12-19$ Yet, the accurate description of the electronic coupling between organic and inorganic materials requires highly demanding ab-initio methods, including electronic correlation. ${ }^{2021}$ Finally, the calculation of charge injection deep inside the organic material requires the simulation of the charge dynamics that could be tackled with a kinetic Monte Carlo approach. 22,26 Nonetheless, an accurate analysis of interface phenomena affecting charge injection is of crucial relevance for a comprehensive description of the working mechanisms of organic electronic devices and is needed in order to screen and guide the synthesis of new compounds or device processes. 122728

The main features of the charge transfer mechanism at the interface between the electrodes and the organic materials can be related to the details of the morphology at the molecular level and to the resulting electronic coupling. The chemical affinity between the molecular material and the electrode determines the strength of the interaction, leading to either weakly- (physisorbed) or strongly-bound (chemisorbed) species. On the basis of the molecule/electrode interaction, formation of nanoscale aggregates is usually observed at the interface. ${ }^{29 \mid 30}$ In turn, morphology controls the electronic coupling between the molecular layer and the electrode, in terms of electronic overlap and energy renormalization of molecular orbitals at the interface due to charge transfer or polarization mechanisms. $31-34$

The formation of the metal-organic interface in organic electronic devices has been the subject of several research efforts focused on the understanding of phenomena related to the generation of the injection current. $\frac{10}{10}$ One of the aspects of the metal-organic interface that has more thoroughly been investigated concerns the development of an energy offset between the Fermi level of metals

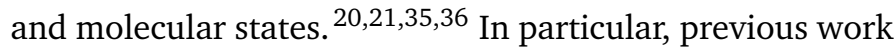
has targeted the investigation of the metal/molecule interaction in model systems or in ordered organic aggregates at the interface with metals, as for example in selfassembled monolayers. ${ }^{920137.40}$ However, the interplay between nanoscale morphology at the interface, electronic coupling and resulting injection rates has not been investigated in detail. Systematic calculations of such complex systems have been made possible only recently thanks to methodological advances and improvements of computing facilities. $41 / 42$

In this work, we analyse the morphology and resulting charge injection properties at the interface between a metallic anode and an organic layer in an electrode/organic junction. Namely, we target hole injection in a typical OLED stack at the interface between indium tin oxide (ITO) and an amorphous undoped thin-film of the Iridium complex Tris[(3-phenyl-1H-benzimidazol-1-yl2(3H)-ylidene)-1,2-phenylene]Ir (DPBIC), commonly used in state-of-the-art phosphorescent OLED devices as hole transport material.12 43/44 Despite its wide use as transparent electrode both in OLEDs and solar cell devices, the properties of the ITO surface and ITO/organic interfaces have been investigated only in few theoretical works. $.45+50$ The understanding of charge injection processes at the metal/organic interface, in complex morphologies, requires an in-depth analysis of intrinsically different phenomena. In our approach, large-scale molecular dynamics (MD) calculations are first used to simulate the morphology of nanoscale aggregates at the metal/organic interface. In particular, a realistic description of interfaces between the metal electrode and amorphous organic aggregates is achieved through the application of an innovative simulation protocol, based on equilibrium and nonequilibrium MD. The resulting configurations are analysed in terms of relevant structural parameters, highlighting the main features of molecular aggregation at the interface. Nanoscale morphologies are then used for the subsequent evaluation of electronic properties and quantitative calculation of the hole transfer rates from the metal to the first layer of adsorbed molecules. In order to achieve this goal, density functional theory (DFT) calculations are used to compute electronic couplings of the metal/organic interface and to evaluate hole hopping rates based on Marcus theory. This computational approach provides a quantitative estimate of the distribution of injection rates for nanoscale aggregates of a realistic electrode/organic interface. This work highlights for the first time the interplay between nanoscale morphology and disorder near the metal interface and its consequences to the statistical vari- 
ations of electronic couplings and hopping rates between the metal and the adsorbed molecules. It is a first step towards a comprehensive in-silico modelling of interfaces, guiding synthesis and processing of organic layers.

\section{Computational details}

Force fields - A non-bonded force-field (FF), based on the Buckingham potential, including polarization terms for oxygen atoms, was applied to $\mathrm{In}_{2} \mathrm{O}_{3}$ and ITO. ${ }^{51}$ This FF provides structural parameters for $\mathrm{In}_{2} \mathrm{O}_{3}$ and ITO bulk supercells in excellent agreement with experimental values (see ESI). 52 Both bonded and non-bonded terms, based on the OPLS-AA force-field,,$\sqrt{53}$ were used to model the DPBIC molecule, as described in previous works. $\frac{12[43}{1}$ The overall RMS deviation of force-field relaxed atomic positions of DPBIC against the DFT optimized geometry is about 0.015 $\mathrm{nm}$ for non-hydrogen atoms. The interaction between DPBIC molecules and the ITO was described in terms of an interaction potential including Lennard-Jones (LJ) and Coulomb parameters. The atomic charges used for the isolated ITO and DPBIC were left unchanged also in the interacting system, keeping consistency with those calculations. The LJ terms for atoms involved in the DPBIC/ITO interaction were taken from the OPLS force field, augmented by terms extracted from the Universal force-field ${ }^{54}$ for ITO metal atoms, applying standard Lorentz-Berthelot rules for cross terms. This force-field provides interaction energies and equilibrium geometries that are in good agreement with values computed at the DFT level, ${ }^{[5]}$ including dispersion corrections (see ESI).

Molecular dynamics - MD calculations were performed by using the GROMACS package. ${ }^{[56}$ A short-range cut-off of $1 \mathrm{~nm}$ was used for Coulomb and van der Waals interactions, including long-range electrostatic terms evaluated via the particle-mesh Ewald (PME) method. ${ }^{[57}$ The Berendsen thermostat ${ }^{58}$ was used for simulations in the NVT (constant volume) ensemble, with a time constant of $0.1 \mathrm{ps,}$ and a Berendsen barostat, ${ }^{58}$ with a time constant of $1.0 \mathrm{ps}$ for simulations in the NPT (constant pressure) ensemble. Periodic boundary conditions (PBCs) in three dimensions were used in all MD calculations, using a large $(\approx 10 \mathrm{~nm})$ vacuum region in the $\mathrm{z}$ direction of the box for simulations of slabs.

DFT relaxations - DFT geometry optimization of $\mathrm{In}_{2} \mathrm{O}_{3} /$ ITO bulk systems were performed by using the Perdew-Burke-Ernzerhof (PBE) gradient-corrected approximation $^{59}$ and Martins-Troullier (MT) pseudopotentials ${ }^{60}$ (taken from http://www.quantum-espresso.org), with a wavefunction cut-off of 80 Ry. Calibration calculations were also performed by using other pseudopotentials and exchange correlation functionals (see ESI). DFT calcula- tions were carried out by using the Quantum Espresso package. 61

Electronic couplings and injection rates - The electronic coupling between ITO and DPBIC molecules was evaluated by computing the transfer integral between ITO Bloch states around the Fermi energy and the molecular HOMO state. In the calculations we restrict the analysis to the HOMO level of DPBIC since the HOMO-1 energy state is about $250 \mathrm{meV}$ below. Since this is about $10 k_{B} T$ at room temperature, the occupation factor makes the hopping rate into this level negligible. The contribution of individual transfer integrals, $H_{i j}$, from the electrode state $i$ into the molecular HOMO state $j$, can be derived in a way similar to the work by Kirkpatrick et al. ${ }^{62}$ The computation involves three independent calculations (DPBIC molecule, ITO slab, and DPBIC@ITO) in order to reconstruct the effective overlap and Hamiltonian matrices. Since the original work was developed for isolated molecules, care must be taken in the $k$-summation of the periodic system. The technical details can be found in the Appendix. Transfer integrals were computed on individual DPBIC/ITO configurations at the interface, extracted from MD, by plane-wave DFT calculations performed with the Quantum Espresso package and applying the PBE exchange-correlation functional, norm-conserving MT pseudopotentials and a wavefunction cut-off of 80 Ry. A plane wave code was chosen in order to accurately capture the wavefunction in the interstitial region between the electrode and physisorbed molecules. The ionic degrees of freedom are approximated within the semiclassical reorganization energy of the Marcus-Jortner approach. ${ }_{63] 64}^{64}$ The Marcus-Jortner approximation has been widely used in the evaluation of charge transfer rates in the hopping regime, especially in the context of simulation of charge transport in weaklycoupled aggregates of organic molecular systems. $31 \mid 65+68$ Hence the transfer rate reads:

$$
R=\frac{2 \pi}{h} \sum_{k}\left|J_{k}\right|^{2}\left[1-f\left(E_{k}\right)\right] \frac{1}{\sqrt{4 \pi k_{B} T \lambda}} \exp \left[-\frac{\left(\varepsilon-E_{k}-\lambda\right)^{2}}{4 k_{B} T \lambda}\right]
$$

where $J_{k}$ is the transfer integral between the molecular HOMO and a metal $k$-state (See equation A.11 in the Appendix), $f(E)$ is the Fermi-Dirac distribution function of the electrode with electro-chemical potential $\mu$, defined as $f(E)=\left\{\exp \left[(E-\mu) / k_{B} T\right]+1\right\}^{-1}, T$ is the temperature and $k_{B}$ is the Boltzmann constant. Calculations were performed at room temperature $(\mathrm{T}=300 \mathrm{~K})$. The value of the reorganization energy $\lambda=68 \mathrm{meV}$ for DPBIC was taken from literature. ${ }^{12}$ The term $\varepsilon-E_{k}$ is the energy difference between the HOMO level and electrode levels, but important corrections arise from polarization of the surrounding 
molecules and the electrode. The latter is the dominant term for the absorbed molecules and can be approximated by the image-charge energy. We can call this renormalized molecular energy level near the metal as $\bar{\varepsilon}_{\text {Номо }}$. We notice that in the limit $\lambda \rightarrow 0$ the gaussian tends to a Dirac-delta function and equation 1 can be approximated to:

$$
R=\frac{2 \pi}{h}|J|^{2}\left[1-f\left(\bar{\varepsilon}_{\text {HOMO }}\right)\right] \rho\left(\bar{\varepsilon}_{\text {HOMO }}-\mu\right),
$$

where $\rho(E)$ represents the density of states (DOS) of the system. This expression makes more manifest the exponential sensitivity of the transfer rate with the energy difference $\bar{\varepsilon}_{\text {НОмо }}-\mu$, i.e. on the energy alignment between molecular states and metal Fermi energy.

Density functional in both LDA or GGA flavors is known to give inaccurate energies and band alignments. ${ }^{69}$ Especially for a molecule/metal interface, there are crucial corrections originating from exchange and correlations contributing among the other to image-charge renormalization. ${ }^{29 / 70}$ A possible approach to take these effects into account is within the GW approximation, ${ }^{71}$ however this method is computationally very demanding, 36 becoming too expensive for the DPBIC/ITO supercell comprising about 400 atoms. This is particularly problematic if statistics of the interface morphology requires repeated calculations on a large number of samples. The energy level alignment between the molecule and the electrode in the Schottky-Mott limit (common vacuum level) $\frac{38172}{3}$ is usually approximated as the difference between the ionization potential of the isolated molecule and the work function of the electrode surface. The measured workfunction of ITO samples ranges between 4 and more than 5 $\mathrm{eV}$, depending on surface processing and treatments, 73,74 whereas the computed ionization potential of DPBIC at the solid state is about $5.3 \mathrm{eV}, 12$ with further lowering that can arise from image charge effects at the metal/organic interface. ${ }^{\sqrt[36]{6}}$ These values lead to effective injection barriers ranging from 0 to more than $1 \mathrm{eV}$. Here, for simplicity, we assume a perfect alignment of the HOMO molecular level with the electrode Fermi level (i.e., $\bar{\varepsilon}_{\text {НОмо }}=\mu$ ). It should be pointed out that the level alignment is of crucial importance for a quantitative prediction of the transfer rates. However, we notice that the exponential term containing $\varepsilon-E_{k}$ is factorized from the transfer matrix element, $J_{k}$, hence the two key parameters entering in equation 1 play an independent role and can be studied separately. Setting $\bar{\varepsilon}_{\text {HOMO }}-\mu=0$ is a way to maximize the role that molecular configurations have on electronic couplings contained in $J_{k}$. For the more usual case of $\bar{\varepsilon}_{\text {НОмО }}<\mu$, charge transfer rates are essentially reduced by a constant exponential factor. Although the general formulae are developed including a $k$ - space summation (see Appendix), the size of the simulation cell allowed us to approximate the transfer integrals at the $\Gamma$ point. As shown in equation 1 , the electronic couplings between molecules at the interface and the metal electrode surface enter directly in the Marcus-Jortner equation for the evaluation of injection rates. However, as demonstrated by several previous works, $11|12| 31|33| 44|67 / 75| 77 \mid$ the electronic coupling changes dramatically with the specific molecule/surface configuration. Therefore, the distribution of electronic couplings with the complex morphology at the interface is a prerequisite for correlating nanoscale molecular aggregation to the distribution of injection rates.

\section{Results and discussion}

\section{Model of the ITO surface}

The thermodynamically stable phase of $\operatorname{In}_{2} \mathrm{O}_{3}$ is a bixbyite body-centered cubic structure, with 40 atoms in the unit cell (80 atoms in the cubic cell), ${ }^{52 \mid 55}$ formally related to a vacant cubic fluorite $\left(\mathrm{CaF}_{2}\right)$ crystal. ${ }^{55 / 78 / 79} \mathrm{An}$ initial model of the $\mathrm{In}_{2} \mathrm{O}_{3}$ bulk crystal was prepared according to available experimental data. $\frac{80}{\mathrm{DFT}}$ calculations were initially carried out on a model of $\operatorname{In}_{2} \mathrm{O}_{3}$, relaxing atom positions and cell parameters in the cubic symmetry (see ESI). At the level of theory used (see Computational Details) the optimized cell parameter (10.047 Å) agrees remarkably well with the experimental value $(10.117 \AA) . \underline{52}$ The computed band gap for $\mathrm{In}_{2} \mathrm{O}_{3}$ is found to be $1.85 \mathrm{eV}$, which is about $1 \mathrm{eV}$ smaller than the value currently accepted. ${ }^{81}$ This discrepancy, however, is ascribed to the well-known underestimation of the band gap related to the use of gradientcorrected XC functionals. Models for the bulk ITO crystal were obtained from the relaxed $\operatorname{In}_{2} \mathrm{O}_{3}$ structures. In the ionically-compensated doping, which is likely to occur at high tin concentrations and oxidizing conditions and is considered to be the most stable in common ITO samples. ${ }^{71}$ indium ions are substituted by $\left(2 \mathrm{Sn}_{I n} \cdot \mathrm{Oi}^{\prime}\right)$ defects, with interstitial oxygen atoms occupying the vacancy sites of $\operatorname{In}_{2} \mathrm{O}_{3} \cdot{ }^{71}$ We focused our simulations on the $5 \mathrm{wt} \%$ Sn-doped systems, in line with common ITO samples. $\underline{52}$ The position of substitutional Sn ions was randomly selected from the metallic sites of the $\operatorname{In}_{2} \mathrm{O}_{3}$ crystal. The electronic structure of ITO bulk models was simulated with the same set-up used for DFT calculations on $\mathrm{In}_{2} \mathrm{O}_{3}$, using a MT pseudopotential for the $\mathrm{Sn}$ sites. The variation in the computed cell side length upon tin doping is below 1\%. From the bulk system, an oxygen-terminated metal slab was obtained by cleaving the cubic crystal along the (111) lowindex surface, which is the most thermodynamically stable. $\frac{55}{5}$ The resulting slab has a hexagonal symmetry and a thickness of about $1 \mathrm{~nm}$, corresponding to 9 atomic layers. The slab was further optimized by DFT, with fixed cell vol- 

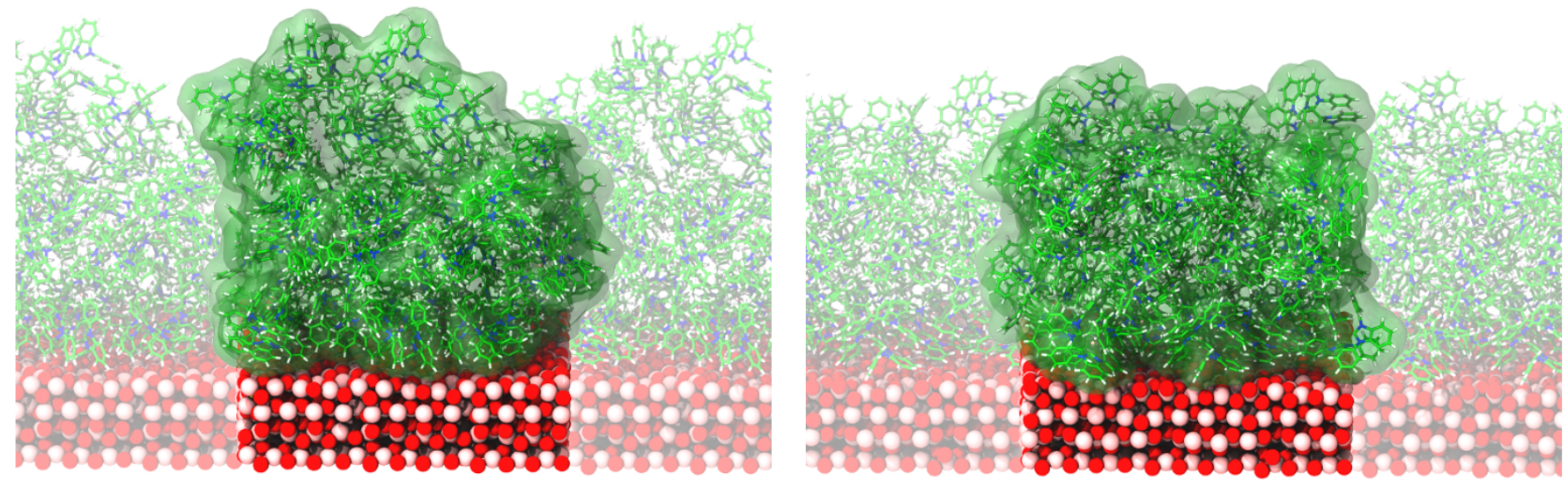

Fig. 1 Representative configurations of kinetic (left) and thermodynamic (right) aggregates of DPBIC molecules on the model ITO surface. Green: carbon; blue: nitrogen; red: oxygen, pink: indium; yellow: tin; white: hydrogen. The shaded area represents periodic images of the simulation box.

ume and constraining the position of the four bottom-most atomic layers (see ESI). From the slab, a 2-dimensional supercell was generated by replicating the DFT unit cell. The size of the slab (5x5 nm) was chosen large enough to allow for subsequent growth of an amorphous organic layer of DPBIC molecules. Moreover, the supercell approach allows a reduction of the slab unit cell symmetry, leading to a change of the angle between the crystal axis from $120^{\circ}$ to $90^{\circ}$. The surface of the ITO slab model can be considered as representative of the exposed nanoscale morphology in polycrystalline ITO thin films, where strong surface local ordering is observed in domains with sizes in the order of a few nanometers. ${ }^{[82} \mathrm{A}$ vacuum region of about $10 \mathrm{~nm}$ was inserted in the direction of the box orthogonal to the ITO surface, to avoid spurious interaction between slab images. The ITO slab supercell was relaxed by constant-volume (NVT) MD simulations under the potential described in the Computational Details section. An annealing/quenching cycle of $1 \mathrm{~ns}$ at a temperature of $500 \mathrm{~K}$ was also performed to improve surface relaxation, followed by equilibration at room temperature.

\section{Morphology of the ITO/DPBIC interface}

The interface morphology between DPBIC and the ITO electrode was simulated by modelling the growth of an amorphous layer of organic molecules onto a relaxed ITO slab, through a combination of non-equilibrium and equilibrium MD. This approach enables the realistic description of complex interface morphologies involving amorphous organic phases and, at the same time, a statistical variability related to the replication of the same thermodynamical systems with different initial conditions. Namely, DP-
BIC molecules were consecutively added to the simulation box and relaxed onto the ITO surface, thus mimicking the growing mechanism in vapor-phase deposition techniques. Despite the simulated growth rate is much faster than real depositions by several orders of magnitude, our approach allows the correlation between growth conditions and morphology by tuning simulation times and annealing temperatures. The formation of the electrode/organic interface was realized by iteratively inserting DPBIC molecules, equilibrated at $300 \mathrm{~K}$, to the $5 \times 5 \mathrm{~nm}$ ITO slab model. Each molecule was initially positioned in the vacuum region above the slab, at a distance of about $10 \mathrm{~nm}$, with a random in-plane displacement and orientation. Initial velocity components of $0.4 \mathrm{~nm} / \mathrm{ps}$, in the -z direction, were added to all atoms of the newly inserted DPBIC, and a short (10 ps) non-equilibrium MD run was performed. In all our simulations, the DPBIC molecules get in contact with the surface within this MD time frame. After each molecule/surface contact, we applied two different annealing protocols. In a first set of simulations, a MD equilibration run of $100 \mathrm{ps}$ at $300 \mathrm{~K}$ was performed for each of the DPBIC molecules. This rather short equilibration time takes to the extreme the condition corresponding to a kinetically-controlled growth mode. In a second set of simulations, a longer MD run of 1 ns was performed between successive molecular insertions, annealing the system with a thermostat with a suitable temperature profile. Specifically, the system was first equilibrated at $300 \mathrm{~K}$ for $200 \mathrm{ps}$, annealed to $500 \mathrm{~K}$ for $100 \mathrm{ps}$, equilibrated at $500 \mathrm{~K}$ for $300 \mathrm{ps}$, quenched to $300 \mathrm{~K}$ for $200 \mathrm{ps}$ and finally equilibrated at $300 \mathrm{~K}$ for $200 \mathrm{ps}$. This annealing strategy mimics molecular relaxations occurring upon application of thermal treatments, due to the higher temperatures and longer 

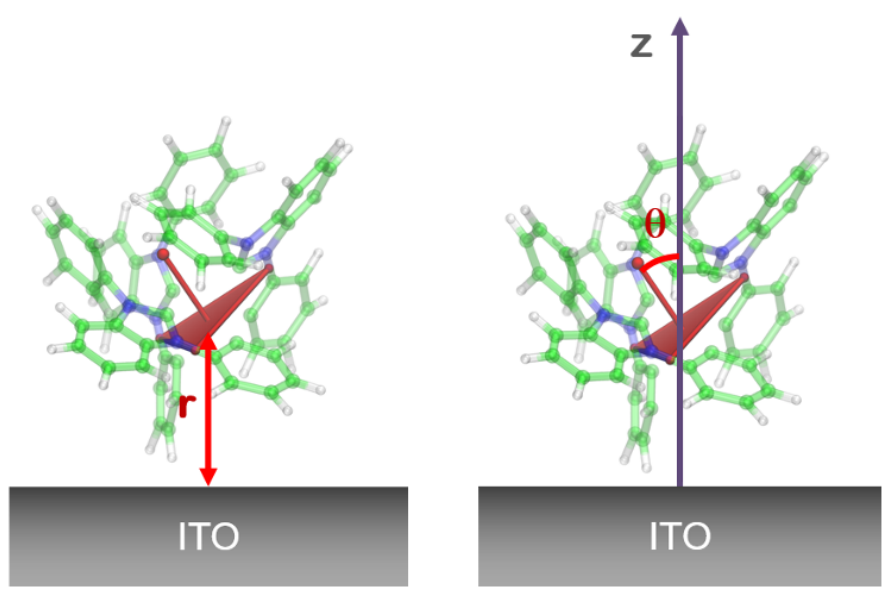

Fig. 2 Radial (left) and angular (right) structural parameters defining the distance and orientation of DPBIC molecules with respect to the ITO surface.

time scales involved. Therefore, this second approach aims at reproducing a thermodynamically-controlled growth of DPBIC aggregates on the ITO surface. Indeed, annealing at high temperature, yet within the experimental stability range (larger than $350{ }^{\circ} \mathrm{C}^{83}$ ) is expected to increment the diffusion of DPBIC molecules on the ITO surface, improving aggregation and molecular packing. For each simulation sequence, about 200 DPBIC molecules were deposited onto the $5 \times 5 \mathrm{~nm}$ ITO model, generating an organic layer with a thickness of about $5 \mathrm{~nm}$. Each simulation process was repeated 50 times with different initial conditions, in order to obtain meaningful statistics of the molecular configurations at the interface. Representative configurations of DPBIC aggregates on ITO for the two growth modes considered are shown in Fig. 1. In order to further gain insights in the simulated morphologies it is convenient to introduce two relevant structural parameters of the DPBIC and ITO interface (see Fig. 2). A first parameter was defined as the distance between the center of mass of the DPBIC and the plane defined by the average $\mathrm{z}$ coordinate of the outermost atoms of the ITO slab. As a further parameter, we considered the orientation angle of DPBIC molecules with respect to the ITO slab, defined as the angle between the normal to the ITO slab (z axis) and the $\mathrm{C}_{3}$ axis of the DPBIC molecule (see Fig. 2p), which also represents the orientation of the molecular static dipole moment. ${ }^{84}$ The global set of morphologies obtained by the MD simulations described above was analysed in terms of these two structural parameters, restricting to molecules near the interface (defined as the molecules with center of mass within a distance of $0.9 \mathrm{~nm}$ from the ITO surface). The resulting distributions of the radial and angular parameters are shown in Fig. 3 for both the kinetically and thermodynamically controlled growth modes. The analy-
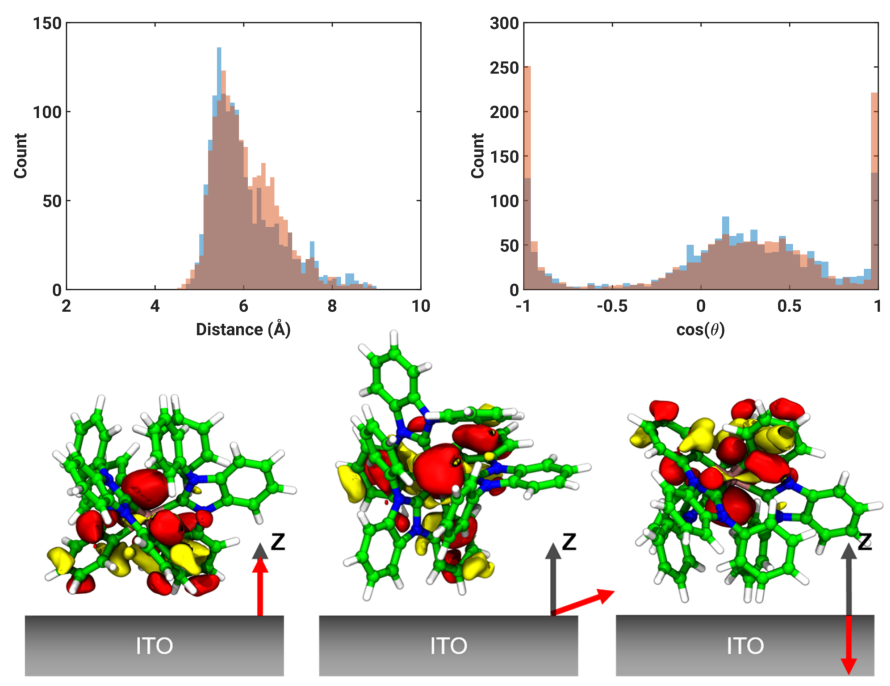

Fig. 3 Histograms (blue: kinetic growth mode; red: thermodynamic growth mode) of the distribution of the DPBIC/ITO distance (top, left) and orientation (top, right), defined in terms of the radial and angular parameters described above, for molecules at the interface. Molecular configuration and $\mathrm{HOMO}$ isosurface for the three most likely orientations $\left(180^{\circ}, 75^{\circ}\right.$ and $0^{\circ}$, from left to right) of DPBIC with respect to the ITO surface (bottom).

sis of the interface configurations suggests a distribution of DPBIC/ITO distances peaked at about $0.6 \mathrm{~nm}$ for both growth modes. This distance is about $0.15 \mathrm{~nm}$ lower than the equilibrium distance for an isolated DPBIC surface on ITO, thus indicating a collective effect of the aggregate in inducing a stronger interaction with the surface. Looking at the distribution of orientation angles, we observe three features, peaked at about $0^{\circ}, 75^{\circ}$, and $180^{\circ}$, respectively. The peaks at $0^{\circ}$ and $180^{\circ}$ are notably sharper than that at $75^{\circ}$, leading to the conclusion that these two orientations are preferred. In the thermodynamic-growth samples these two angular orientations are even more favored, from which we conclude that they are the most energetically stable. The two most favored orientations of DPBIC molecules at the interface with ITO are also highlighted by the two main peaks in the ITO/DPBIC distance distribution, with a main feature located at about $0.57 \mathrm{~nm}$ (parallel orientation with respect to the z-axis, see Fig. 3) and a second shoulder at about $0.65 \mathrm{~nm}$ (antiparallel orientation). The occurrence of two main preferential orientation and a third broader distribution of orientations can be ascribed to the peculiar symmetry of the DPBIC, owing to both its electrostatics and geometrical shape, where the first is likely to be the dominating cause. ${ }^{84}$ Nevertheless, despite the occurrence of preferential geometries, the molecular configurations at the interface can potentially assume different values in a quite broad set of values, as a result of the disorder induced by the formation of amorphous aggregates. As discussed above, small changes in the molecule/surface 

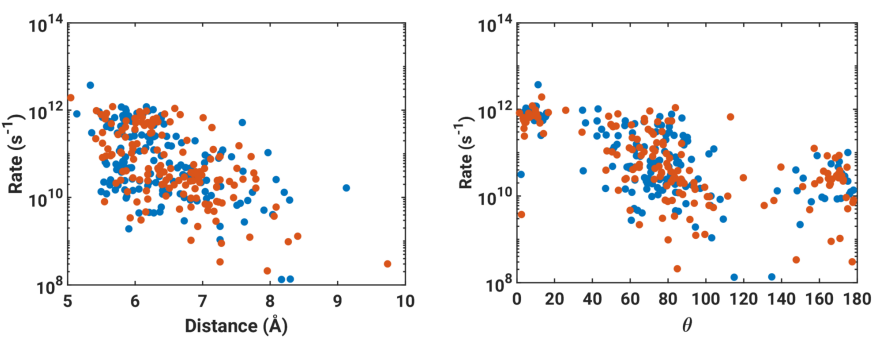

Fig. 4 Variation of the injection rate as a function of the DPBIC/ITO distance (left) and orientation (right) for the kinetic (blue dots) and thermodynamic (red dots) growth modes, respectively.

geometry can impact greatly on the injection rates. Therefore, the distribution of molecular configurations at the interface, as it will be explained below, can be related to the resulting distribution of injection rates.

\section{Electronic couplings and injection rates}

The electronic couplings between DPBIC molecules and ITO were computed on representative sample configurations extracted from the whole set of simulations on the basis of the statistical distributions discussed in the previous section, according to their statistical frequencies. A grid of 10x10 points for the radial and angular parameters $(r, \theta)$ was used, resulting in a total of 100 individual configurations. For each pair of values $\left(r_{i}, \theta_{i}\right)$ on this grid, a representative DPBIC/ITO system with the values $(r, \theta)$ closest to the target parameters was selected from the whole set of samples. This approach allows to extract the configuration of a single molecule in the realistic environment of aggregated (equilibrated) morphologies, and in contact with the ITO surface, on a specific site. For the subsequent quantum-mechanical calculation, the DPBIC/ITO subsystem was isolated and projected on a periodic cell with the hexagonal periodicity of the ITO slab. It is worth recalling that the ITO slab, used in MD simulations for the growth of the organic layer, was built as a supercell of the DFT model, and constant-volume MD equilibrations were only performed, thus keeping the cell parameters unchanged. Consequently, the re-cut operation applied to the MD model leads to a slab with the same periodicity of the initial DFT model. For each configuration considered, transfer integrals and injection rates were computed as described in the Computational Details section. The resulting distribution of hopping rates, from the ITO slab to the DPBIC molecules, in terms of the radial and angular structural parameters, is shown in Fig. 4. Not surprisingly, the computed transfer integrals exhibit an exponential decay as the distance between the DPBIC molecule and the ITO surface increases, which is directly related to the exponential wavefunction decay. The most interesting
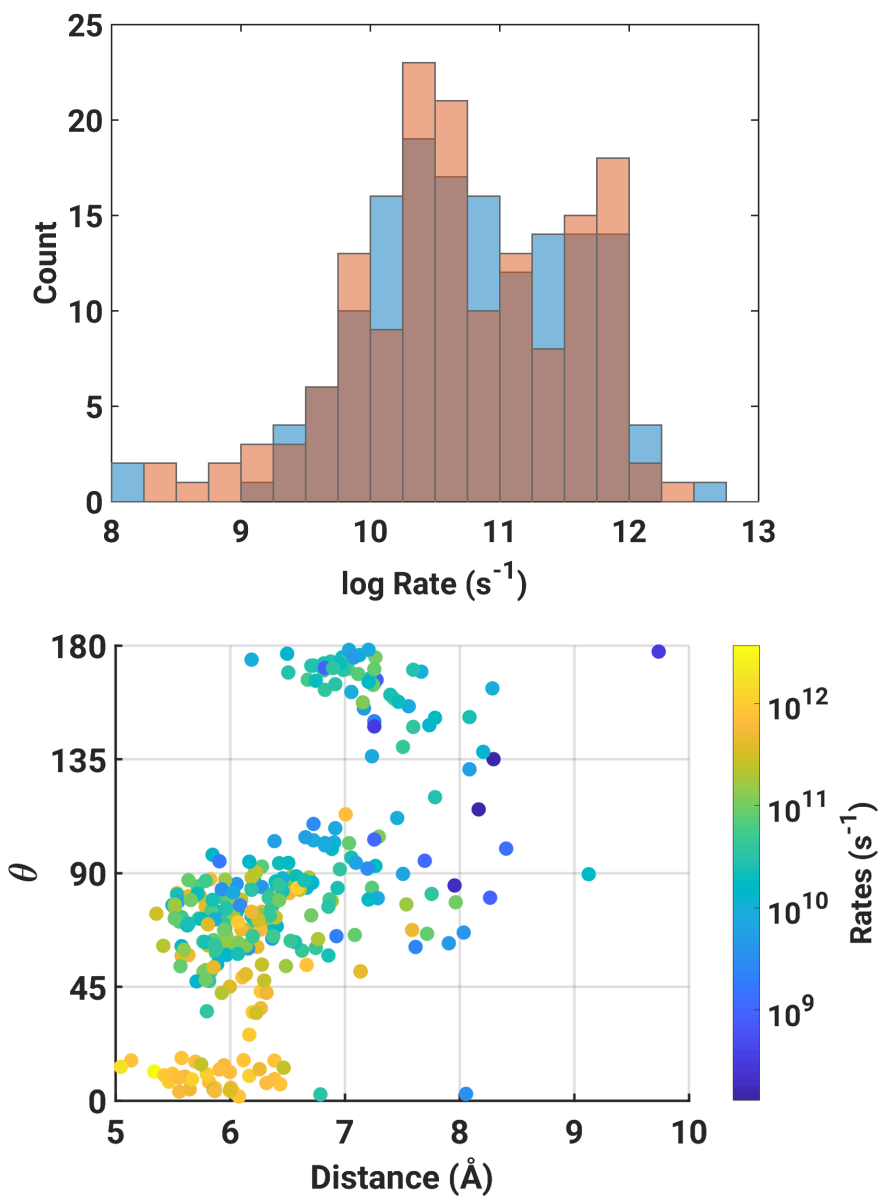

Fig. 5 Distribution of injection rates (top) in kinetic (blue bars) and thermodynamic (red bars) growth conditions and distribution of injection rates, expressed as hopping frequencies in $\mathrm{Hz}$ (bottom, log scale), as a function of distance and orientation for the aggregated (kinetic and thermodynamic growth) data.

behavior is shown with the angular dependence (Fig 4p). Computed values suggest a preferential hole injection into molecules oriented around $\theta=0^{\circ}$, for which hopping rates as high as $5 \cdot 10^{12} s^{-1}$ have been estimated. For the opposite $\left(\theta=180^{\circ}\right)$ orientation the rate decreases by more than one order of magnitude. Clearly this is related to the orientation of the HOMO molecular orbital, as shown in Fig. 3 Most of the population related to the peak around $\theta=75^{\circ}$ exhibits a rather broad scattering of rates, ranging over 3 orders of magnitudes.

The overall distribution of hopping rates is shown in Fig. 5. The largest fraction of computed injection rates lies in the range from $10^{10}$ to $10^{12} \mathrm{~Hz}$, in line with rates computed for other physisorbed metal-organic systems,, 76 as in the case of the weakly coupled DPBIC and ITO. It is worth recalling, however, that the absolute values of the injection rates are computed by assuming a perfect level alignment (i.e., null initial barrier) between the involved molecular energy level and the electrochemical potential of the 
electrode, thus representing an upper limit to the effective injection rate. Computed injection rates show a slightly more peaked distribution for DPBIC molecules grown in thermodynamic conditions, with respect to the kineticallycontrolled growth mode, although the differences are not very pronounced. The two-dimensional diagram in Fig. 5 shows also the correlation between injection rates and both radial and angular structural parameters, obtained using all the available samples. The distribution suggests some preferential configurations for optimal injection.

As expected, the largest values of computed injection rates are observed for the shortest DPBIC-ITO distances correlated with molecules oriented at about $0^{\circ}$ with respect to the surface. However, orientation of individual molecules does also play an important role, with a significant fraction of molecules at the interfaces oriented at $75^{\circ}$ and located at distances between 0.60 and $0.65 \mathrm{~nm}$ and contributing with large hopping rates. It is worth noting that the hopping of a charge from the ITO surface into adsorbed DPBIC molecules is the first step of a complex injection process, involving the percolation of charge carriers deep inside the organic materials. Nevertheless, a detailed knowledge on the distribution of hopping rates at the metal-organic interface constitutes a prerequisite for investigations on the charge transport mechanisms in complex materials and devices. We plan to devote this analysis to a future study.

\section{Conclusions}

In summary, a computational approach for coupling molecular morphology to charge transfer properties at the electrode/organic interface was presented and applied to the case of thin films of DPBIC, an iridium-based emitter and hole transport material for OLEDs, on ITO. The potential role of materials processing and, in particular, heat treatments was assessed by comparing kinetic with thermodynamic growth conditions, simulated by different MD protocols. Simulations suggest a strong relationship between molecular orientation, related to the symmetry of the HOMO molecular level, and charge transfer properties at the interfaces. The resulting distribution of rates, for a hole hopping from the ITO into an adsorbed DPBIC molecule, is centered at about a few tens of $\mathrm{THz}$, assuming a perfect energy level alignment, and spans more than two orders of magnitude, depending on the specific configuration at the interface. The correlation between interface morphology and computed hopping rates suggests a significant role of the electrode/molecule coupling, in addition to the involved energy barriers, in determining the overall efficiency of the charge injection mechanism. Targeting strategic materials in the development of OLEDs, the ap- proach outlined in this work is general and can be extended to other materials and interfaces of interest involved in the injection of charges from a metal electrode into an organic layer. The intimate connection between molecular structure, morphology in molecular aggregates at interfaces and charge transport, highlighted in this study, underlines the need for investigations of nanoscale phenomena for a detailed understanding of the working mechanism of complex functional devices based on organic materials.

\section{Acknowledgements}

We acknowledge the CINECA Supercomputing Center (Italy) award under the ISCRA initiative, for the availability of high performance computing resources and support. This work was supported by the project "MOSTOPHOS Modeling stability of organic phosphorescent light emitting diodes" of the NMP-20-2014 H2020 programme under Grant Agreement No. 646259.

\section{Appendix}

For the calculation of the transfer rate we apply the Fermi golden rule which is also at the basis of the semiclassical Marcus-Jortner formula. ${ }^{64}$ The initial and final states are generally not orthogonal. As shown in Baumeier et al., 62 however, it is possible to perform three separate calculations in order to reconstruct the overlap and the effective matrix element. The aim here is to extend that formalism to plane wave approaches. To this end, as suggested by Ruhle et al., 85 we perform three separate calculations of the eigenstates and energy levels of the isolated metal, isolated molecule and coupled system. The calculation has been implemented as a post-processing of the plane-wave code Quantum Espresso (QE). ${ }^{61}$ Across the three calculations we take care that the supercell used and cutoff are the same, such that the number of Fourier coefficients are the same and all projections are consistent. This is certainly true if we restrict to a $\Gamma$-point approximation. When $k$-sampling is allowed, further considerations are needed (see below). We compute the transfer rate from a localized molecular level, $\phi_{m}^{i}(r)$, to a Bloch state, $\phi_{k n}^{f}(r)$. These can be represented in plane waves as:

$$
\begin{gathered}
\phi_{m}^{i}(r)=\sum_{G} C_{m}^{i}(G) e^{i G r} \\
\phi_{k n}^{f}(r)=\sum_{G^{\prime}} C_{k n}^{f}\left(G^{\prime}\right) e^{i\left(k+G^{\prime}\right) r}
\end{gathered}
$$

The Bloch states of the coupled system,

$$
\psi_{k^{\prime} l}(r)=\sum_{G^{\prime \prime}} C_{k^{\prime} l}^{f}\left(G^{\prime \prime}\right) e^{i\left(k^{\prime}+G^{\prime \prime}\right) r}
$$


form a complete basis set on which the states of the isolated sub-units can be projected. What is needed is just a complete set to represent the envelope of the wave functions and this can be obtained at any $k$-point of equation (A.3). For simplicity we choose $k=0$, hence using this complete basis set the Hamiltonian can be represented as

$$
H=\sum_{l}\left|\psi_{0 l}>H<\psi_{0 l}\right|
$$

We note the above directly relates to the Luttinger states. ${ }^{86}$ Therefore, all the needed projections can be computed from the following expressions:

$$
\begin{gathered}
\varepsilon_{i}=<\phi_{m}^{i}|H| \phi_{m}^{i}>=\sum_{l} \sum_{G, G^{\prime}} C_{m}^{i *}(G) C_{l}(G) \varepsilon_{0 l} C_{l}^{*}\left(G^{\prime}\right) C_{m}^{i}\left(G^{\prime}\right) \\
\varepsilon_{f}=<\phi_{k n}^{f}|H| \phi_{k n}^{f}>=\sum_{l} \sum_{G, G^{\prime}} C_{k n}^{f *}(G) C_{l}(G) \varepsilon_{0 l} C_{l}^{*}\left(G^{\prime}\right) C_{k n}^{f}\left(G^{\prime}\right) \\
H_{i f}=<\phi_{m}^{i}|H| \phi_{k n}^{f}>=\sum_{l} \sum_{G, G^{\prime}} C_{m}^{f *}(G) C_{l}(G) \varepsilon_{0 l} C_{l}^{*}\left(G^{\prime}\right) C_{k n}^{f}\left(G^{\prime}\right) \\
S_{i f}=<\phi_{m}^{i} \mid \phi_{k n}^{f}>=\sum_{l} \sum_{G, G^{\prime}} C_{m}^{f *}(G) C_{l}(G) C_{l}^{*}\left(G^{\prime}\right) C_{k n}^{f}\left(G^{\prime}\right)
\end{gathered}
$$

Note that terms like the overlap matrix, $S_{i f}$, are generally complex, but they can be made purely real by adjusting the arbitrary phase of the initial and final states. Then, it is possible to construct an orthogonal basis, needed for the correct application of the Fermi golden rule, with the help of the following transformations (with $S=S_{i f}, S^{+}=$ $\sqrt{1-S}+\sqrt{1+S}$ and $S^{-}=\sqrt{1-S}-\sqrt{1+S}$,

$$
\begin{gathered}
\mid 1>=\frac{1}{2 \sqrt{1-S^{2}}}\left[S^{+}\left|\phi^{i}>+S^{-}\right| \phi^{f}>\right] \\
\mid 2>=\frac{1}{2 \sqrt{1-S^{2}}}\left[S^{-}\left|\phi^{i}>+S^{+}\right| \phi^{f}>\right]
\end{gathered}
$$

Note that for $S \rightarrow 0$ we recover the correct limits $\mid 1>\rightarrow$ $\mid \phi^{i}>$ and $|2>\rightarrow| \phi^{f}>$. The effective transfer integral is then obtained as,

$$
H_{12}=<1|H| 2>=\frac{1}{1-S^{2}}\left[H_{i f}-S_{i f} \frac{\varepsilon_{i}+\varepsilon_{f}}{2}\right]
$$

This last equation corresponds to $J_{k}$ of equation 1 .

A final remark concerns the $G$-summations of equations (A.5-8). Here the Fourier coefficients must be summed consistently between the three calculations. Since Quantum Espresso stores the coefficients $C(G)$ for increasing values of $|k+G|$ rather than $|G|$, they must be reordered when the calculations are not at the $\Gamma$-point. For this purpose, the code $p w 2 g w . f 90$, a filter distributed with the QE package, was used.

\section{References}

1 C. E. Petoukhoff, D. K. Vijapurapu and D. M. O'Carroll, Solar Energy Materials and Solar Cells, 2014, 120, 572583.

2 M. Vasilopoulou, A. M. Douvas, D. G. Georgiadou, V. Constantoudis, D. Davazoglou, S. Kennou, L. C. Palilis, D. Daphnomili, A. G. Coutsolelos and P. Argitis, Nano Research, 2014, 7, 679-693.

3 P. Tyagi, R. Srivastava, L. I. Giri, S. Tuli and C. Lee, Synthetic Metals, 2016, 216, 40-50.

4 Q. H. Wu, Critical Reviews in Solid State and Materials Sciences, 2013, 38, 318-352.

5 N. R. Armstrong, P. A. Veneman, E. Ratcliff, D. Placencia and M. Brumbach, Accounts of Chemical Research, 2009, 42, 1748-1757.

6 J.-H. Kim, R. E. Triambulo and J.-W. Park, Journal of Applied Physics, 2017, 121, 105304.

7 C. Hinzmann, O. Magen, Y. J. Hofstetter, P. E. Hopkinson, N. Tessler and Y. Vaynzof, ACS Applied Materials \& Interfaces, 2017, 9, 6220-6227.

8 D. Natali and M. Caironi, Advanced Materials, 2012, 24, 1357-1387.

9 G. Heimel, L. Romaner, E. Zojer and J. L. Bredas, Nano Letters, 2007, 7, 932-940.

10 J. C. Scott, Journal of Vacuum Science \& Technology A: Vacuum, Surfaces, and Films, 2003, 21, 521-531.

11 V. Coropceanu, H. Li, P. Winget, L. Zhu and J.-L. Brédas, Annual Review of Materials Research, 2013, 43, 6387.

12 P. Kordt, J. J. M. van der Holst, M. Al Helwi, W. Kowalsky, F. May, A. Badinski, C. Lennartz and D. Andrienko, Advanced Functional Materials, 2015, 25, 1955-1971.

13 M. Mesta, M. Carvelli, R. J. De Vries, H. Van Eersel, J. J. Van Der Holst, M. Schober, M. Furno, B. Lüssem, K. Leo, P. Loebl, R. Coehoorn and P. A. Bobbert, Nature Materials, 2013, 12, 652-658.

14 L. Viani, C. Risko, M. F. Toney, D. W. Breiby and J. L. Brédas, ACS Nano, 2014, 8, 690-700.

$15 \mathrm{M}$. Yoneya, H. Minemawari, T. Yamada and T. Hasegawa, Journal of Physical Chemistry C, 2017, 121, 8796-8803.

16 M. Kratzer and C. Teichert, Nanotechnology, 2016, 27, 292001.

17 O. M. Roscioni, L. Muccioli, R. G. Della Valle, A. Pizzirusso, M. Ricci and C. Zannoni, Langmuir, 2013, 29, 8950-8958.

18 A. Lorenzoni, M. Muccini and F. Mercuri, Journal of Physical Chemistry C, 2017, 121, 21857-21864.

19 A. Lorenzoni, F. Gallino, M. Muccini and F. Mercuri, RSC Adv., 2016, 6, 40724-40730. 
20 J. B. Neaton, M. S. Hybertsen and S. G. Louie, Physical Review Letters, 2006, 97, 1-4.

21 A. Biller, I. Tamblyn, J. B. Neaton and L. Kronik, Journal of Chemical Physics, 2011, 135, 164706.

22 R. G. E. Kimber, E. N. Wright, S. E. J. O'Kane, A. B. Walker and J. C. Blakesley, Physical Review B, 2012, 86, 235206.

23 R. Coehoorn, H. Van Eersel, P. A. Bobbert and R. A. Janssen, Advanced Functional Materials, 2015, 25, 2024-2037.

24 G. Gryn'ova, A. Nicolaï, A. Prlj, P. Ollitrault, D. Andrienko and C. Corminboeuf, J. Mater. Chem. C, 2017, 5, 350-361.

25 A. Lorenzoni, M. Muccini and F. Mercuri, RSC Advances, 2015, 5, 11797-11805.

26 J. Cottaar, R. Coehoorn and P. Bobbert, Organic Electronics, 2012, 13, 667-672.

27 F. Steiner, C. Poelking, D. Niedzialek, D. Andrienko and J. Nelson, Phys. Chem. Chem. Phys., 2017, 19, 1085410862.

28 D. Beljonne, J. Cornil, L. Muccioli, C. Zannoni, J. L. Brédas and F. Castet, Chemistry of Materials, 2011, 23, 591-609.

29 S. Braun, W. R. Salaneck and M. Fahlman, Advanced Materials, 2009, 21, 1450-1472.

30 H. Ma, H.-L. Yip, F. Huang and A. K.-Y. Jen, Advanced Functional Materials, 2010, 20, 1371-1388.

31 J. Zhao, M. Feng, D. B. Dougherty, H. Sun and H. Petek, ACS Nano, 2014, 8, 10988-10997.

32 N. Armbrust, F. Schiller, J. Güdde and U. Höfer, Scientific Reports, 2017, 7, 1-8.

33 J. Hwang, A. Wan and A. Kahn, Materials Science and Engineering R: Reports, 2009, 64, 1-31.

34 N. Koch, N. Ueno, A. Thye and S. Wee, The MoleculeMetal Interface, WILEY-VCH Verlag, 2013, p. 272.

35 O. T. Hofmann, P. Rinke, M. Scheffler and G. Heimel, ACS Nano, 2015, 9, 5391-5404.

36 D. A. Egger, Z. F. Liu, J. B. Neaton and L. Kronik, Nano Letters, 2015, 15, 2448-2455.

37 K. S. Thygesen and A. Rubio, Physical Review Letters, 2009, 102, 1-4.

38 N. Koch, Journal of Physics Condensed Matter, 2008, 20, 184008.

39 G. Heimel, L. Romaner, E. Zojer and J. L. Bredas, Accounts of Chemical Research, 2008, 41, 721-729.

40 I. Campbell, J. Kress and R. Martin, Applied Physics Letters, 1997, 71, 3528-3530.

41 N. Martsinovich and A. Troisi, Physical Chemistry Chemical Physics, 2012, 14, 13392.
42 D. R. Jones and A. Troisi, Physical Chemistry Chemical Physics, 2010, 12, 4625.

43 P. Kordt and D. Andrienko, Journal of Chemical Theory and Computation, 2016, 12, 36-40.

44 P. Kordt, S. Stodtmann, A. Badinski, M. Al Helwi, C. Lennartz and D. Andrienko, Phys. Chem. Chem. Phys., 2015, 17, 22778-22783.

45 P. B. Paramonov, S. A. Paniagua, P. J. Hotchkiss, S. C. Jones, N. R. Armstrong, S. R. Marder and J. L. Bredas, Chemistry of Materials, 2008, 20, 5131-5133.

46 E. L. Hanson, J. Guo, N. Koch, J. Schwartz and S. L. Bernasek, Journal of the American Chemical Society, 2005, 127, 10058-10062.

47 P. J. Hotchkiss, H. Li, P. B. Paramonov, S. A. Paniagua, S. C. Jones, N. R. Armstrong, J. L. Brédas and S. R. Marder, Advanced Materials, 2009, 21, 4496-4501.

48 H. Li, P. Paramonov and J.-L. Bredas, Journal of Materials Chemistry, 2010, 20, 2630.

49 Y. Lee, H. Lee, S. Park and Y. Yi, Applied Physics Letters, 2012, 101, 233305.

50 M. Gliboff, H. Li, K. M. Knesting, A. J. Giordano, D. Nordlund, G. T. Seidler, J. L. Brédas, S. R. Marder and D. S. Ginger, Journal of Physical Chemistry C, 2013, 117, 15139-15147.

51 R. A. Buckingham, Proceedings of the Royal Society A, 1938, 168, 264-283.

52 O. Warschkow, D. E. Ellis, G. B. Gonzalez and T. O. Mason, Journal of the American Ceramic Society, 2003, 86, 1700-1706.

53 W. L. Jorgensen, D. S. Maxwell and J. Tirado-Rives, Journal of the American Chemical Society, 1996, 118, 11225-11236.

54 A. K. Rappé, C. J. Casewit, K. S. Colwell, W. A. Goddard and W. M. Skiff, Journal of the American Chemical Society, 1992, 114, 10024-10035.

55 A. Walsh and R. A. Catlow, Journal of Materials Chemistry, 2010, 20, 10438-10444.

56 D. Van Der Spoel, E. Lindahl, B. Hess, G. Groenhof, A. E. Mark and H. J. Berendsen, Journal of Computational Chemistry, 2005, 26, 1701-1718.

57 T. Darden, D. York and L. Pedersen, The Journal of Chemical Physics, 1993, 98, 10089-10092.

58 H. J. C. Berendsen, J. P. M. Postma, W. F. van Gunsteren, A. DiNola and J. R. Haak, The Journal of Chemical Physics, 1984, 81, 3684-3690.

59 A. D. Becke, The Journal of Chemical Physics, 1993, 98, 5648-5652.

60 N. Troullier and J. L. Martins, Physical Review B, 1991, 43, 8861-8869.

61 P. Giannozzi, S. Baroni, N. Bonini, M. Calandra, R. Car, 
C. Cavazzoni, D. Ceresoli, G. L. Chiarotti, M. Cococcioni, I. Dabo, A. Dal Corso, S. De Gironcoli, S. Fabris, G. Fratesi, R. Gebauer, U. Gerstmann, C. Gougoussis, A. Kokalj, M. Lazzeri, L. Martin-Samos, N. Marzari, F. Mauri, R. Mazzarello, S. Paolini, A. Pasquarello, L. Paulatto, C. Sbraccia, S. Scandolo, G. Sclauzero, A. P. Seitsonen, A. Smogunov, P. Umari and R. M. Wentzcovitch, Journal of Physics Condensed Matter, 2009, 21, 395502.

62 B. Baumeier, J. Kirkpatrick and D. Andrienko, Physical Chemistry Chemical Physics, 2010, 12, 11103.

63 A. Nitzan, Chemical Dynamics in Condensed Phases, Oxford University Press, 2006.

64 V. May and O. Kühn, Charge and energy transfer dynamics in molecular systems, WILEY-VCH Verlag, 2011.

65 J. Idé, R. Méreau, L. Ducasse, F. Castet, Y. Olivier, N. Martinelli, J. Cornil and D. Beljonne, Journal of Physical Chemistry B, 2011, 115, 5593-5603.

66 A. Troisi, Chemical Society Reviews, 2011, 40, 2347.

67 G. Pourtois, D. Beljonne, J. Cornil, M. A. Ratner and J. L. Brdas, Science, 2002, 1, 4436-4447.

68 D. Veldman, S. Chopin, S. Meskers and R. Janssen, The Journal of Physical Chemistry A, 2008, 112, 8617 âĂş8632.

69 F. Flores, J. Ortega and H. Vázquez, Physical Chemistry Chemical Physics, 2009, 11, 8658.

70 H. Vázquez, Y. J. Dappe, J. Ortega and F. Flores, Journal of Chemical Physics, 2007, 126, 144703.

71 G. Strinati, H. J. Mattausch and W. Hanke, Physical Review Letters, 1980, 45, 290-294.

72 S. Braun, W. Osikowicz, Y. Wang and W. R. Salaneck, Organic Electronics: physics, materials, applications, 2007, 8, 14-20.

73 J. S. Kim, M. Granström, R. H. Friend, N. Johansson, W. R. Salaneck, R. Daik, W. J. Feast and F. Cacialli,
Journal of Applied Physics, 1998, 84, 6859-6870.

74 Y. Gassenbauer and A. Klein, Journal of Physical Chemistry $B, 2006,110,4793-4801$.

75 A. Massé, P. Friederich, F. Symalla, F. Liu, R. Nitsche, R. Coehoorn, W. Wenzel and P. A. Bobbert, Physical Review B, 2016, 93, 195209.

76 J. Wiberg, T. Marinado, D. P. Hagberg, L. Sun, A. Hagfeldt and B. Albinsson, The journal of physical chemistry. B, 2010, 114, 14358-63.

77 V. Coropceanu, J. Cornil, D. A. da Silva Filho, Y. Olivier, R. Silbey and J. L. Brédas, Chemical Reviews, 2007, 107, 926-952.

78 J. Rosen and O. Warschkow, Physical Review B - Condensed Matter and Materials Physics, 2009, 80, 1-10.

79 G. B. Gonzalez, J. B. Cohen, J. H. Hwang, T. O. Mason, J. P. Hodges and J. D. Jorgensen, Journal of Applied Physics, 2001, 89, 2550-2555.

80 M. Marezio, Acta Crystallographica, 1966, 20, 723-728.

81 A. Walsh, J. L. F. Da Silva, S. H. Wei, C. Korber, A. Klein, L. F. J. Piper, A. Demasi, K. E. Smith, G. Panaccione, P. Torelli, D. J. Payne, A. Bourlange and R. G. Egdell, Physical Review Letters, 2008, 100, 2-5.

82 U. Betz, M. Kharrazi Olsson, J. Marthy, M. F. Escolá and F. Atamny, Surface and Coatings Technology, 2006, 200, 5751-5759.

83 Luminescence Technology Corp., Lumtec Product Sheet LTN658, http://www. Iumtec.com.tw.

84 P. Friederich, R. Coehoorn and W. Wenzel, Chemistry of Materials, 2017, 29, 9528-9535.

85 V. Rühle, A. Lukyanov, F. May, M. Schrader, T. Vehoff, J. Kirkpatrick, B. Baumeier and D. Andrienko, Journal of Chemical Theory and Computation, 2011, 7, 33353345.

86 J. M. Luttinger and W. Kohn, Physical Review, 1955, 97, 869. 